\title{
RADIATIVE TRANSFER IN DYNAMIC STELLAR \\ ATMOSPHERES
}

\author{
RICHARD I. KLEIN \\ National Center for Atmospheric Research, Boulder, Colo., U.S.A.
}

\begin{abstract}
In order to understand the Be star phenomenon, and move in directions away from ad hoc models, detailed calculations have been made that treat nonlinear hydrodynamics self-consistently with non-L.T.E. radiative transfer.
\end{abstract}

We investigate the radiating shock wave produced by a constant-velocity piston moving into an atmosphere in radiative, hydrostatic and statistical equilibrium. Self-consistent numerical solutions are obtained to the equations of hydrodynamics, radiative transfer and level population. Only Lyman continuum radiation is considered, although calculations including the transfer of radiation in higher continua are underway. The results are interpreted in terms of the relaxation lengths for the collisional and radiative processes, and by comparing the 'radiative' case both with one in which only collisional transitions occur and with the adiabatic case.

The shock quickly develops a temperature spike. At large optical depth, the structure of the spike is controlled by collisional ionization and three-body recombination. When the shock approaches $\tau_{912} \approx 209$, precursor radiation drives an ionization front rapidly through the remaining atmosphere. Preliminary calculations for multi-continuum radiative transfer indicate a radiation precursor effect from the shock front when the shock is much deeper in the atmosphere at earlier times. This is a result of the gas becoming optically thin to photons of frequencies very different from those in the Lyman continuum. At small optical depth, escape of recombination radiation narrows the temperature spike producing a radiation dominated shock. Long collisional recombination times compared to gas travel times in the upper atmosphere produce a large plateau of nearly constant ionization behind the shock.

\section{DISCUSSION}

Sonneborn: In a star rotating very rapidly near breakup velocity, if you hit it hard enough with a shock, could you get mass ejection?

Klein: This is quite possible but our models do not include the effects of rotation.

Cardona: Is it possible to include the effects of rotation in your formulation?

Klein: The inclusion of rotation is difficult on two levels: in the hydrodynamics and in the radiative transfer. In the latter case it is not clear that the problem can be done, given the finite constraints of the 7600 computer. The hope for a model which includes both the hydrodynamics and the radiative transfer is therefore a long way off.

Noerdlinger: To study ejection of matter you would probably want to include radiation force in your first equation. The shock could well thin out the material in the upper layers to the point where radiation pressure, especially in the lines, is important.

Klein: Absolutely.

Haisch: In your calculations what takes the place of the piston in a real star?

Klein: In the Be stars radiation pressure might act as a self-starting mechanism. 